in the interventional suite. Color-coded MIP images enable rapid diagnosis and interpretation of large multiphasic CTA data.

Disclosures K. Narsinh: None. K. Mueller: 5; C; Siemens Healthineers. M. Manhart: 5; C; Siemens Healthineers. S. Hetts: 1; C; Siemens Healthineers, NIH. 2; C; Imperative Medical, MicroVention Terumo, Route 92 Medical. T. Moore: None. E. Chaney: None. D. Cooke: 1; C; Siemens Healthineers.

\section{E-042 CAN HEMATOLOGICAL INDICES DIFFERENTIATE BETWEEN STROKE VS. STROKE MIMICS? A RETROSPECTIVE, SINGLE INSTITUTION ANALYSIS}

${ }^{1} \mathrm{G}$ Dawod*, ${ }^{1} \mathrm{~N}$ Henkel, ${ }^{1} \mathrm{~N}$ Karim, ${ }^{1} \mathrm{H}$ Salahuddin, ${ }^{2} \mathrm{~S}$ Shawver, ${ }^{2} \mathrm{~S}$ Zaidi, ${ }^{2} \mathrm{M}$ Jumaa. ${ }^{1}$ Neurology, University of Toledo Medical Center, Toledo, OH; ${ }^{2}$ Neurology, Promedica Toledo Hospital, Toledo, $\mathrm{OH}$

\subsection{6/neurintsurg-2020-SNIS.78}

Introduction/Purpose In the past five years, red blood cell distribution width (RDW) values have been used in cardiovascular research to predict outcomes in patients with atherosclerotic disease. Since large vessel occlusion (LVO) ischemic strokes share a similar pathogenesis to acute coronary artery syndromes, recent studies have attempted to elucidate the relationship between RDW values and acute ischemic strokes (AIS). Thus far, the studies have shown an association between AIS and an increased RDW. We explored this relationship in a subset of patients with a severe neurological presentation at our institution to better understand the clinical significance of RDW in various cerebrovascular pathologies.

Materials and Methods Patients with Rapid Arterial oCclusion Evaluation (RACE) scores $>4$ were used for this analysis. A retrospective analysis of hematological indices was undertaken with IRB approval. Complete blood counts (CBCs), complete metabolic panels (CMPs), and coagulation studies drawn at the time of ED arrival were reviewed for each patient. For statistical analysis, multi-way ANOVA, t-Tests, and Chi-square analyses were conducted in ' $R$ ' to assess these indices across different diagnostic groups.

Results This study included 492 patients with pre-hospital RACE scores $>4$ as identified by EMS in the pre-hospital 
setting. Final diagnoses were the following: ischemic stroke (IS) without large vessel occlusion (LVO) - 14.8\%; IS with LVO - 37\%; TIA - 6.5\%, ICH - 13.8\%; stroke mimics $27.9 \%$. We separately analyzed red cell distribution width (RDW), neutrophil count, lymphocyte count, mean corpuscular hemoglobin $(\mathrm{MCH})$, platelet to lymphocyte ratios, and neutrophil to lymphocyte ratios. Patients who suffered from any cerebrovascular accident (CVA) had a lower RDW than mimics $(p=0.02)$. Further analysis revealed RDW to be significantly decreased in the ICH cohort $(p=0.02)$ compared to the stroke mimics, but did not differ from the other diagnoses. When controlling for anticoagulation status or gender, RDW and the platelet-lymphocyte ratios were not significantly different across diagnoses.

Conclusion Although recent studies have implicated a significant relationship between an increased RDW and the occurrence of AIS, our study does not support this finding. Contrarily, with a larger cohort than in those of previous retrospective studies, we found patients with acute cerebral infarcts across all ages to have significantly lower RDW values when compared with stroke mimics. Efforts for the identification of a reliable hematologic biomarker in addition to RACE scores for the timely diagnosis of ischemic stroke remains a priority in the initial pre-admission work-up. CBCs remain rapid and cost-effective tools upon admission, and should be further analyzed to identify other hematologic indices that can aid in the diagnosis of ischemic stroke. Further studies are warranted to completely define how blood tests are implicated in post-stroke outcomes.

Disclosures G. Dawod: None. N. Henkel: None. N. Karim: None. H. Salahuddin: None. J. Shawver: None. S. Zaidi: None. M. Jumaa: None.

\section{E-043 AUTOMATED LARGE VESSEL OCCLUSION BY ARTIFICIAL INTELLIGENCE IMPROVES STROKE WORKFLOW METRICS: 1ST 100 PATIENT EXPERIENCE IN A HUB AND SPOKE STROKE SYSTEM}

1,2D Dornbos, III* 1,2D Hoit, 1,2 V Inoa-Acosta, 1,2C Nickele, 1,2 A Arthur, 1,2 L Elijovich ${ }^{1}$ Neurological Surgery, Semmes-Murphey Clinic, Memphis, TN; ${ }^{2}$ Neurological Surgery, University of Tennessee Health Science Center, Memphis, TN

\subsection{6/neurintsurg-2020-SNIS.79}

Introduction Large vessel occlusion (LVO) acute ischemic stroke is a time sensitive disease process. Technology that could shorten the time to mechanical thrombectomy (MT) holds great promise, but experience and data on these applications are sparse. We describe our initial experience with Artificial Intelligence (AI) for automated LVO detection (aLVOd) in a hub and spoke network.

Methods This is a retrospective chart review of consecutive code stroke cases at a comprehensive stroke center and two spoke hospitals. Viz AI was implemented from May 2019 to December 2019, during which time 100 consecutive LVO alerts were evaluated. Baseline clinical characteristics were collected on all aLVO alerts and from historical LVO controls taken to the angiography suite for intervention from the 6 months prior to AI implementation. Details of medical treatment, CT/CTA interpretation by blinded Neuroradiologists (confirmed LVO, normal, or significant cerebrovascular finding without LVO), final diagnosis, results of MT, clinical outcomes, and stroke workflow metrics were collected.
Categorical variables were compared with Pearson chi-squared testing and continuous variables with Wilcoxon rank sum testing.

Results A total of 680 code strokes were evaluated during the AI study period. The average age and NIHSS score of the 100 aLVOd patients were 69 (IQR 57, 80) and 4 (IQR 1, 13.75), respectively. There were no significant differences in baseline characteristics of LVO patients identified by aLVOd, those LVOs missed by aLVOd, or historical LVO controls undergoing MT. A total of 68 patients were diagnosed with LVO during the AI study period; 45 (66.2\%) were identified by aLVOd and confirmed by neuroradiology, and 23 (33.8\%) were missed by AI. The majority of LVOs missed by AI were distal M2 or posterior circulation occlusions (60.8\%). Of the 100 total aLVOd alerts, 55 were not LVO based on the neuroradiologist interpretation (18 were normal, 36 high grade intracranial atherosclerosis, and one was non-diagnostic). The average NIHSS score of LVO patients detected by AI was 11 (IQR 3, 19). Forty-one patients during the AI study period underwent MT and 36 historical LVO controls underwent mechanical thrombectomy.

The sensitivity and specificity of aLVOd was $66.2 \%$ and 91\% while the positive predictive value and negative predictive values of AI were $45 \%$ and $96 \%$, respectively. Median time from CTA completion to treating team notification for all patients was significantly shorter for aLVOd patients (8 minutes (IQR 4.25, 9) v $17 \mathrm{~min}$ (IQR 13, 28.5), p<0.001). Door to arterial puncture was also significantly faster for patients transferred from a spoke to a hub for MT (141 minutes (IQR 128.5, 168) v $185 \mathrm{~min}$ (IQR 151, 241), $\mathrm{p}=0.027)$.

Conclusion VizAI aLVOd is a robust screening tool for identifying LVO based on its high negative predictive value. This system is a valuable adjunct to traditional imaging interpretation. The use of AI to detect LVO improves stroke workflow metrics in a hub and spoke stroke system of care.

Disclosures D. Dornbos: None. D. Hoit: None. V. InoaAcosta: None. C. Nickele: None. A. Arthur: None. L. Elijovich: None.

\section{E-044 CAPITATED PRICING MODEL FOR STROKE THROMBECTOMIES: A SINGLE INSTITUTION EXPERIENCE}

${ }^{1} \mathrm{~K}$ Shah* ${ }^{2} \mathrm{M}$ Brown, ${ }^{1} \mathrm{~S}$ Desai, ${ }^{3} \mathrm{~B}$ Jankowitz, ${ }^{4} \mathrm{~T}$ Jovin, A Jadhav, $15213^{5}$, B Gross, $15213^{6} .{ }^{1}$ Neurology, University of Pittsburgh Medical Center, Pittsburgh, PA; ${ }^{2}$ Neurology and Neurosurgery, Louisiana State University, New Orleans, LA; ${ }^{3}$ Neurosurgery, Cooper University Health Care, Camden, NJ; ${ }^{4}$ Neurology and Neurosurgery, Cooper University Health Care, Camden, NJ; ${ }^{5}$ Neurology and Neurosurgery, University of Pittsburgh Medical Center, Pittsburgh, PA; ${ }^{6}$ Neurosurgery, University of Pittsburgh Medical Center, Pittsburgh, PA

\subsection{6/neurintsurg-2020-SNIS.80}

Introduction With a continued rise in health care expenditures, there is a demonstrable focus on curbing expenses. Recent studies estimate direct and indirect costs of stroke to be $\$ 73.7$ - 103.5 billion $^{1,2}$. Since 2015, numerous studies demonstrate the benefit of mechanical thrombectomy (MT) for treatment of large vessel occlusions (LVOs) ${ }^{3-10}$; however, notable costs are associated with devices utilized in each procedure. Some institutions have negotiated capitated pricing models in an effort to reduce these costs; however, the cost savings has yet to be critically evaluated. 